[Chem. Pharm. Bull.

$[35(2) 647-653(1987)]$

\title{
Synthesis and Biological Activity of Fluorine-Modified Platelet Activating Factors ${ }^{1)}$
}

\author{
Kagari Fujita, ${ }^{a}$ Susumu Kobayashi, ${ }^{a}$ IChiro Kudo, ${ }^{a}$ Keizo Inoue, ${ }^{a}$ \\ Shoshichi Nojima, ${ }^{a}$ Masaji Ohno, ${ }^{*}$ Yoshiro Kobayashi, ${ }^{*}, b$ \\ MAKoto OdaGiRi, ${ }^{b}$ and TAKeO TAGUCHI ${ }^{b}$ \\ Faculty of Pharmaceutical Sciences, University of Tokyo, ${ }^{a}$ Hongo, Bunkyo-ku, \\ Tokyo 113, Japan and Tokyo College of Pharmacy, ${ }^{b}$ Horinouchi, \\ Hachioji, Tokyo 192-03, Japan
}

(Received July 14, 1986)

\begin{abstract}
Fluorine-modified acetyl glyceryl ether phosphorylcholines (platelet activating factors; PAFs) were efficiently synthesized in an enantioselective manner by the coupling of a D-threitol.derivative with fluorinated long-chain alkoxy methanesulfonates. The introduction of a trifluoromethyl group at the terminal of the alkyl ether chain decreased the hypotensive activity and platelet activation considerably. As the number of fluorine atoms in the alkyl ether chain was increased, both activities were gradually restored, but no selective agonist was obtained from among the fluorinated PAFs.
\end{abstract}

Keywords_ platelet activating factor; fluorine-modified platelet activating factor; D-threitol derivative; alkylation

Since the characterization of platelet activating factors (PAFs) (1a, 1b) by Hanahan et al., ${ }^{2)}$ and Benveniste et al., ${ }^{3)}$ in 1979, this new class of biologically active lipids has been the subject of a great deal of synthetic study in the field of medicinal chemistry. They act as powerful mediators in physiological or pathological processes such as anaphylaxis and inflammation. ${ }^{4)}$ We reported in a previous communication ${ }^{5)}$ an efficient methodology for the preparation of both enantiomers of $\mathrm{C}_{16^{-}}$and $\mathrm{C}_{18}$-PAFs, starting from D- and/or L-tartaric acids as chiral synthons, and found that the unnatural PAFs possess far lower biological activities than the natural ones. Some analogues with modifications of the hydrophilic quaternary ammonium moiety were also synthesized and their biological activities were investigated. The modification of the cationic moiety of PAF caused a large diminution or enhancement of both biological activities (platelet activation and hypotension), in the same direction.

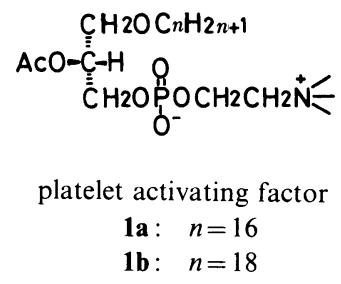

We were next interested in modification of the lipophilic alkyl moiety, especially the introduction of fluorine atoms into the chain. In general, introduction of fluorines into biologically active compounds is expected to change the properties of the parent hydrogensubstituted compounds in the direction of enhanced lipophilicity and metabolic stability 
without appreciable change of steric bulkiness. ${ }^{6,7)}$

Thus, to examine the substituent effect of fluorine on the biological activities of PAF, some analogues having fluorine atoms in the $1-O$-alkyl chain were synthesized and their biological activities were investigated.

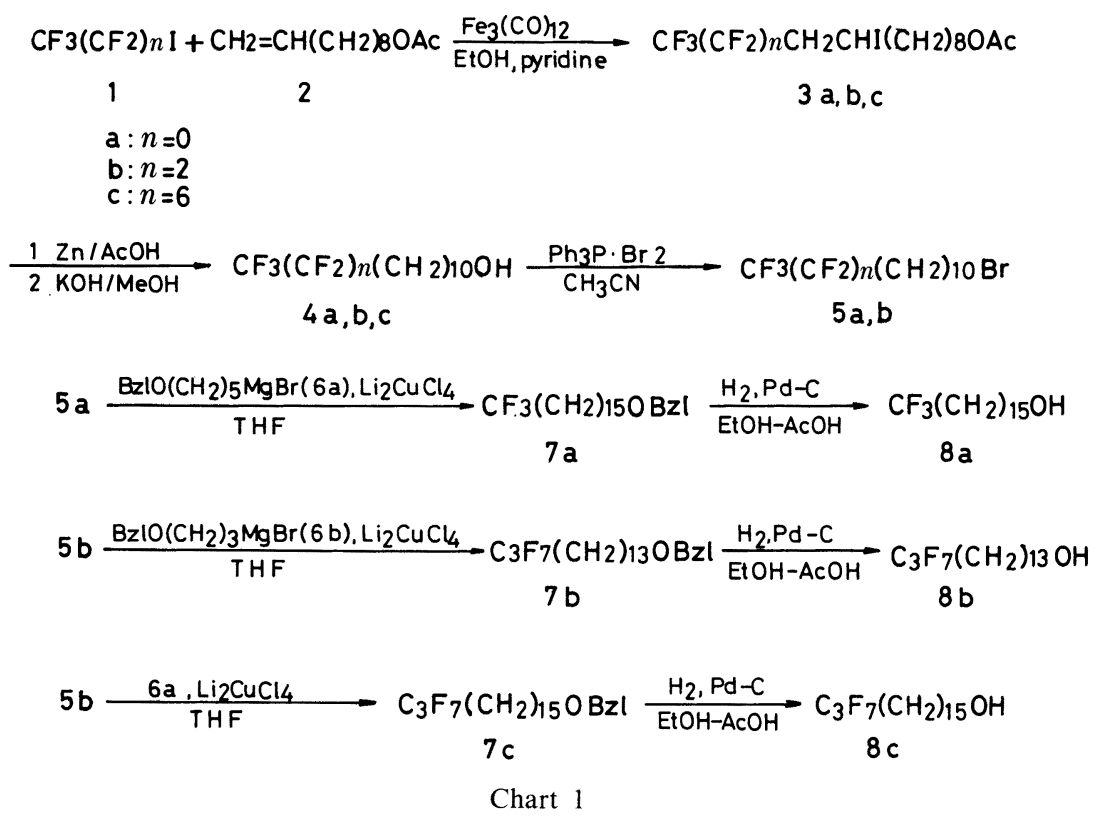

Synthesis of Fluorinated $\mathrm{C}_{16}-\mathrm{C}_{18}$ Alcohols (Chart 1)

The $\mathrm{C}_{16}-\mathrm{C}_{18}$ alcohols $(\mathbf{4} \mathbf{c}$ and $\mathbf{8 a}-\mathbf{c})$ were prepared as follows. The radical addition reaction of perfluoroalkyl iodide $(\mathbf{1 a}-\mathbf{c})$ to the terminal olefinic compound (2) catalyzed by
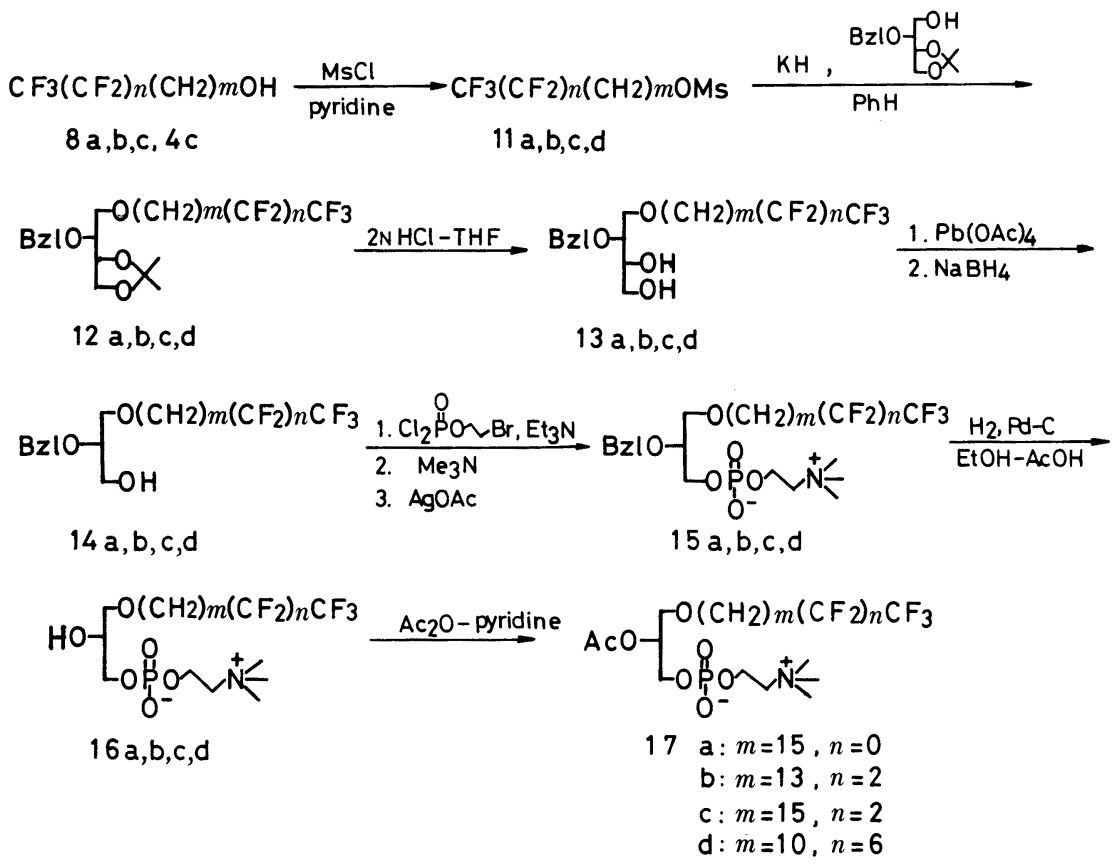

Chart 2 
$\mathrm{Fe}_{3}(\mathrm{CO})_{12}{ }^{8}$ afforded the adducts $(3 \mathbf{a}-\mathbf{c})$, which were in turn reduced with $\mathrm{Zn}$ dust in acetic acid followed by saponification to give the fluorinated alcohols $(\mathbf{4 a}-\mathbf{c})$. The Grignard coupling reaction of $\omega$-benzyloxyalkyl magnesium bromide $(\mathbf{6 a}, \mathbf{b})$ with the bromides $(\mathbf{5 a}, \mathbf{b})$ derived from $4 \mathbf{a}$ and $\mathbf{4 b}$ proceeded in the presence of a catalytic amount of $\mathrm{Li}_{2} \mathrm{CuCl}_{4}{ }^{9}$ ) to give the benzyl ethers $(\mathbf{7 a}-\mathbf{c})$, which were converted to the $\mathrm{C}_{16}-\mathrm{C}_{18}$ alcohols $(\mathbf{8 a}-\mathbf{c})$ by debenzylation $\left(\mathrm{H}_{2} / \mathrm{Pd}-\mathrm{C}\right)$.

\section{Synthesis of 1-O-Fluoroalkyl-2- $O$-acetyl-sn-glycero-3-phosphocholines (Fluorine-Modified} PAFs) (Chart 2)

The $\mathrm{C}_{16}-\mathrm{C}_{18}$ alcohols were converted to the methanesulfonyl esters and coupled with 2$O$-benzyl-3,4- $O$-isopropylidene-D-threitol, and then converted to 1- $O$-fluoroalkyl-2- $O$-benzyl$s n$-glycerol following the methods described in the previous communication. ${ }^{5)}$

Then phosphorylcholine groups were introduced into the $s n$-3-position of the glycerols, and the benzyl groups were removed and acetylated to afford fluorine-modified PAFs.

\section{Biological Activities of Fluorine-Modified PAFs (Table I)}

The biological activities (platelet activation and hypotensive) of fluorine-modified PAFs were investigated. The results are summarized in Table I, showing the relative biological activities of the analogues. Their ability to induce irreversible platelet aggregation was determined by measuring aggregation and the release of ${ }^{14} \mathrm{C}$-serotonin from rabbit platelets ${ }^{10}$ ) and their hypotensive activities were examined by using male Wistar strain rats. ${ }^{11)}$ The activities of the analogues are expressed relative to that of $\mathrm{C}_{16}$-PAF (reciprocals of the concentrations required).

The results showed that no selective analogue with high hypotensive activity but with low platelet activation was present among the modified PAFs with fluorinated alkyl ether chains. However, it should be noted that the introduction of a trifluoromethyl group at the terminal of the alkyl ether chain (17a) decreased both activities considerably, and as the number of fluorine atoms in the alkyl ether chain was increased, both activities were gradually restored $(\mathbf{1 7 b}, \mathbf{1 7 c}, \mathbf{1 7 d})$. Although the results obtained here are difficult to explain in terms of lipophilicity and metabolic stability, the structure-activity relationships of PAF should be explored further, not only from the viewpoint of constitutional modification, but also by conformational analysis. ${ }^{2)}$

TABLE I. Biological Activities of Fluorine-Modified PAFs

\begin{tabular}{ccc}
\hline \hline & \multicolumn{2}{c}{ Relative activity } \\
\cline { 2 - 3 } & Platelet activation & Hypotension \\
\hline $\mathrm{C}_{16}-\mathrm{PAF}$ & 1 & 1 \\
17a & 0.10 & 0.10 \\
17b & 0.33 & 0.33 \\
17c & 0.58 & 1 \\
17d & 0.63 & 0.30 \\
\hline
\end{tabular}

\section{Experimental}

All the reactions were performed under an argon atmosphere unless otherwise specified, using a standard syringe technique for the transfer of materials. The solvents were generally redistilled before use. Tetrahydrofuran (THF) and ether were dried over sodium and distilled before use. Thin-layer chromatography (TLC) was performed on Merck $60 \mathrm{~F}_{254}(0.25 \mathrm{~mm})$ sheets, and the spots were visualized with molybdophosphoric acids in sulfuric acid. Wakogel C-200 was employed for the column chromatography. Melting points were taken on a hot-stage microscope (Yanagimoto) and are uncorrected. Infrared (IR) spectra were recorded using a Jasco IRA-1 spectrophotometer. 
Proton nuclear magnetic resonance $\left({ }^{1} \mathrm{H}-\mathrm{NMR}\right)$ spectra were recorded on a Varian EM $390 \mathrm{~L}$ instrument and the chemical shifts are reported in parts per million ( $\mathrm{ppm}$ ) relative to tetramethylsilane. ${ }^{19} \mathrm{~F}$-NMR spectra were recorded on a Varian EM 360L spectrometer and the chemical shifts are reported in parts per million relative to benzotrifluoride as an internal standard. The optical rotations were measured with a JASCO DIP 140 polarimeter. The mass spectra (MS) were recorded with a JEOL JMS-DX 300 apparatus. Perfluoroalkyl iodides were purchased from PCR Inc., Gainesville, Florida.

11,11,11-Trifluoroundecan-1-ol (4a)—A $30 \mathrm{ml}$ test-tube containing 9-decenyl acetate $(6.0 \mathrm{~g}, 30.3 \mathrm{mmol})$, ethanol $(3 \mathrm{ml})$, pyridine $(0.9 \mathrm{ml})$ and $\mathrm{Fe}_{3}(\mathrm{CO})_{12}(150 \mathrm{mg})$ was placed in a stainless-steel autoclave, and trifluoromethyl iodide $(7.42 \mathrm{ml}, 89 \mathrm{mmol})$ was introduced. The autoclave was heated at $80^{\circ} \mathrm{C}$ for $2 \mathrm{~h}$, then at $110^{\circ} \mathrm{C}$ for $16 \mathrm{~h}$. The reaction mixture was diluted with ether, washed successively with $1 \mathrm{~N} \mathrm{HCl}$ and brine, then dried over $\mathrm{MgSO}_{4}$. $\mathrm{After}$ removal of the solvent in vacuo, the residue was treated with $\mathrm{Zn}$ powder $(6.53 \mathrm{~g})$ in a mixture of acetic acid $(60 \mathrm{ml})$ and ether $(60 \mathrm{ml})$ at room temperature for $15 \mathrm{~h}$. The precipitate was filtered off through Celite and washed with ether. The filtrate was washed with $1 \mathrm{~N} \mathrm{HCl}, \mathrm{NaHCO}_{3}$ solution and brine, then dried over $\mathrm{MgSO}_{4}$. The solvent was removed in vacuo to leave crude $11,11,11$-trifluoroundecanyl acetate $(5.95 \mathrm{~g})$, which was saponified by treatment with $4.3 \%$ methanolic $\mathrm{KOH}(50 \mathrm{ml})$ at room temperature for $2 \mathrm{~h}$. The reaction mixture was evaporated to remove the solvent and the residue was diluted with water. This was extracted with ether, then the extract was dried over $\mathrm{MgSO}_{4}$ and concentrated in vacuo. The residue was submitted to column chromatography $\left(\mathrm{SiO}_{2}, 10 \%\right.$ ethereal hexane as the eluent) to give 11,11,11-trifluoroundecan-1-ol $(4 \mathrm{a}, 2.556 \mathrm{~g}, 37 \%)$ as a colorless oil. $4 \mathrm{a}:{ }^{1} \mathrm{H}-\mathrm{NMR}\left(\mathrm{CDCl}_{3}\right) \delta: 3.63(2 \mathrm{H}$, $\left.\mathrm{t}, J=6 \mathrm{~Hz},-\mathrm{CH}_{2} \mathrm{OH}\right) .{ }^{19} \mathrm{~F}-\mathrm{NMR}\left(\mathrm{CDCl}_{3}\right) \delta:-3.67(\mathrm{t}, J=9.4 \mathrm{~Hz})$. IR (neat): $3300,2920,2850,1270,1140 \mathrm{~cm}^{-1} . \mathrm{MS}$ $m / z: 208\left(\mathrm{M}^{+}-\mathrm{H}_{2} \mathrm{O}\right), 180,166,152,138,97,83,69$. High-resolution MS Calcd for $\mathrm{C}_{11} \mathrm{H}_{19} \mathrm{~F}_{3}$ : 208.14438. Found: 208.14448 .

1-Bromo-11,11,11-trifluoroundecane (5a)_A dimethylformamide (DMF) solution (10 ml) of $4 \mathrm{a}(2.056 \mathrm{~g}$, $8.7 \mathrm{mmol}$ ) was added to a suspension of triphenylphosphine dibromide [prepared from triphenylphosphine $(3.10 \mathrm{~g}$, $11.8 \mathrm{mmol})$ and bromine $(1.746 \mathrm{~g}, 10.9 \mathrm{mmol})]$ in acetonitrile $(10 \mathrm{ml})$ at $0^{\circ} \mathrm{C}$. The reaction mixture was stirred for $12 \mathrm{~h}$ at room temperature, diluted with water and extracted with ether. The organic layer was washed with water, dried over $\mathrm{MgSO}_{4}$, and then chromatographed $\left(\mathrm{SiO}_{2}, 10 \%\right.$ ethereal hexane) to give the bromide $(5 \mathrm{a}, 2.465 \mathrm{~g}, 94 \%)$ as a colorless oil. 5a: ${ }^{1} \mathrm{H}-\mathrm{NMR}\left(\mathrm{CDCl}_{3}\right) \delta: 3.10\left(2 \mathrm{H}, \mathrm{t}, J=7 \mathrm{~Hz},-\mathrm{CH}_{2} \mathrm{Br}\right)$. MS m/z: $290 \mathrm{M}^{+}\left({ }^{81} \mathrm{Br}\right), 288 \mathrm{M}^{+}\left({ }^{79} \mathrm{Br}\right), 209$, 135, 133. High-resolution MS Calcd for $\mathrm{C}_{11} \mathrm{H}_{20} \mathrm{BrF}_{3}: 289.0699$. Found: 289.0692 .

1-Benzyloxy-16,16,16-trifluorohexadecane (7a)_-A $0.1 \mathrm{M}$ THF solution of $\mathrm{Li}_{2} \mathrm{CuCl}_{4}(0.5 \mathrm{ml})$ was added to a solution of the Grignard reagent [prepared from 5-benzyloxypentyl bromide (780 $\mathrm{mg})$ and $\mathrm{Mg}(100 \mathrm{mg})$ ] in THF $(10 \mathrm{ml})$, followed by the addition of the bromide $5 \mathrm{a}(300 \mathrm{mg}, 1.04 \mathrm{mmol})$. The reaction mixture was stirred for $8 \mathrm{~h}$ at room temperature. After the usual extractive work-up (ether for extraction), the extract was purified by column chromatography $\left(\mathrm{SiO}_{2}, 0.5 \%\right.$ AcOEt in hexane) to give the coupling product $7 \mathbf{a}(350 \mathrm{mg}, 88 \%)$ as a colorless oil. $7 \mathbf{a}$ : ${ }^{1} \mathrm{H}-\mathrm{NMR}\left(\mathrm{CDCl}_{3}\right) \delta: 3.50\left(2 \mathrm{H}, \mathrm{m},-\mathrm{CH}_{2} \mathrm{OBn}\right), 4.53\left(2 \mathrm{H}, \mathrm{s},-\mathrm{OCH}_{2} \mathrm{Ph}\right), 7.37(5 \mathrm{H}, \mathrm{s}, \mathrm{Ph}) .{ }^{19} \mathrm{~F}-\mathrm{NMR}\left(\mathrm{CDCl}_{3}\right) \delta:-3.3$ $(\mathrm{t}, J=11.3 \mathrm{~Hz})$. MS $m / z: 386\left(\mathrm{M}^{+}\right), 295,276$. High-resolution MS Calcd for $\mathrm{C}_{23} \mathrm{H}_{37} \mathrm{~F}_{3} \mathrm{O}: 386.2793$. Found: 386.2781 . IR (neat): $3030,2935,2860,1100 \mathrm{~cm}^{-1}$.

16,16,16-Trifluorohexadecan-1-ol (8a) - Hydrogenolysis of the benzyl ether 7 a $(291 \mathrm{mg}, 0.75 \mathrm{mmol})$ on $5 \%$ $\mathrm{Pd}-\mathrm{C}$ in $\mathrm{EtOH}(5 \mathrm{ml})$ under a pressure of $3 \mathrm{~atm}$ at room temperature followed by recrystallization from $n$-hexane gave the alcohol 8a $(156 \mathrm{mg}, 70 \%)$ as colorless crystals. 8a: $\mathrm{mp} 56-56.5^{\circ} \mathrm{C} .{ }^{1} \mathrm{H}-\mathrm{NMR}\left(\mathrm{CDCl}_{3}\right) \delta: 1.17-1.85(26 \mathrm{H}, \mathrm{m})$, $1.85(1 \mathrm{H}, \mathrm{s},-\mathrm{OH}), 1.80-2.40\left(2 \mathrm{H}, \mathrm{m}, \mathrm{CF}_{3} \mathrm{CH}_{2}-\right), 3.65\left(2 \mathrm{H}, \mathrm{t}, J=5 \mathrm{~Hz},-\mathrm{CH}_{2} \mathrm{O}-\right) .{ }^{19} \mathrm{~F}-\mathrm{NMR}\left(\mathrm{CDCl}_{3}\right) \delta:-3.8(\mathrm{t}, J=$ $10.3 \mathrm{~Hz})$. IR $(\mathrm{KBr}): 3360,2918,2842,1155 \mathrm{~cm}^{-1}$. MS $m / z: 278\left(\mathrm{M}^{+}-18\right), 250$. High-resolution MS Calcd for $\mathrm{C}_{16} \mathrm{H}_{29} \mathrm{~F}_{3}: 278.2219$. Found: 278.2202 .

$\mathbf{1 1 , 1 1 , 1 2 , 1 2 , 1 3 , 1 3 , 1 3 - H e p t a f l u o r o t r i d e c a n - 1 - o l ~ ( 4 b ) - A ~ m i x t u r e ~ o f ~ h e p t a f l u o r o - 1 - i o d o p r o p a n e ~}(6.694 \mathrm{~g}$, $22.6 \mathrm{mmol})$, 9-decenyl acetate $(3.775 \mathrm{~g}, 19 \mathrm{mmol})$ and $\mathrm{Fe}_{3}(\mathrm{CO})_{12}(100 \mathrm{mg})$ in a Pyrex glass sealed tube was heated at $80^{\circ} \mathrm{C}$ for $5.5 \mathrm{~h}$. The reaction mixture was dissolved in a mixture of acetic acid $(33 \mathrm{ml})$ and ether $(33 \mathrm{ml})$, and treated with $\mathrm{Zn}$ powder $(1.95 \mathrm{~g})$ at room temperature for $1 \mathrm{~h}$. Extractive work-up (ether for extraction) followed by chromatographic purification $\left(\mathrm{SiO}_{2}, 20 \%\right.$ ethereal pentane) gave crude heptafluorotridecanyl acetate $(5.268 \mathrm{~g}, 76 \%)$, which was saponified by treatment with $5 \%$ methanolic $\mathrm{KOH}(35 \mathrm{ml})$ at room temperature for $2 \mathrm{~h}$ to give $4 \mathrm{~b}(4.569 \mathrm{~g}$, $71 \%$ overall yield) as a colorless oil. $4 \mathrm{~b}$; bp $135-142{ }^{\circ} \mathrm{C} / 4 \mathrm{~mm}$ (Kugelröhr). ${ }^{1} \mathrm{H}-\mathrm{NMR}\left(\mathrm{CDCl}_{3}\right) \delta: 3.69(2 \mathrm{H}, \mathrm{t}, J=$ $\left.6 \mathrm{~Hz},-\mathrm{CH}_{2} \mathrm{OH}\right) .{ }^{19} \mathrm{~F}-\mathrm{NMR}\left(\mathrm{CDCl}_{3}\right) \delta:-18.1(3 \mathrm{~F}, \mathrm{t}, J=9.4 \mathrm{~Hz}),-52.5(2 \mathrm{~F}, \mathrm{~m}),-65.1(2 \mathrm{~F}, \mathrm{~m}) . \mathrm{IR}$ (neat): $3300 \mathrm{~cm}^{-1}$. MS $m / z: 308\left(\mathrm{M}^{+}-18\right), 280$.

1-Bromo-11,11,12,12,13,13,13-heptafluorotridecane (5b) - By the same method as used for the preparation of 5a, 5b was obtained in $85 \%$ yield. 5b: Colorless oil. ${ }^{1} \mathrm{H}-\mathrm{NMR}\left(\mathrm{CDCl}_{3}\right) \delta: 3.43\left(2 \mathrm{H}, \mathrm{t}, \mathrm{J}=7 \mathrm{~Hz},-\mathrm{CH}_{2} \mathrm{Br}\right) .{ }^{19} \mathrm{~F}-\mathrm{NMR}$ $\left(\mathrm{CDCl}_{3}\right) \delta:-17.3(3 \mathrm{~F}, \mathrm{t}, J=9.4 \mathrm{~Hz}),-52.3(2 \mathrm{~F}, \mathrm{~m}),-65.0(2 \mathrm{~F}, \mathrm{~m})$. IR (neat): $2920,2850,1225,1170 \mathrm{~cm}^{-1} . \mathrm{MS} / z$ : $390 \mathrm{M}^{+}\left({ }^{81} \mathrm{Br}\right), 388 \mathrm{M}^{+}\left({ }^{79} \mathrm{Br}\right)$.

1-Benzyloxy-14,14,15,15,16,16,16-heptafluorohexadecane $(7 \mathrm{~b})$ _- In the same manner as described for the preparation of $\mathbf{7 a}$, the reaction of the Grignard reagent [prepared from 3-benzyloxypropyl bromide $(3.054 \mathrm{~g}$, $13.3 \mathrm{mmol})$ and $\mathrm{Mg}(470 \mathrm{mg}, 20 \mathrm{mmol})]$ with $5 \mathrm{~b}(1.577 \mathrm{~g}, 4.1 \mathrm{mmol})$ in the presence of $\mathrm{Li}_{2} \mathrm{CuCl}_{4}(0.05 \mathrm{mmol})$ in THF at room temperature for $5 \mathrm{~h}$ gave the benzyl ether $7 \mathbf{b}(742 \mathrm{mg}, 40 \%)$ as a colorless oil. $7 \mathbf{b}:{ }^{1} \mathrm{H}-\mathrm{NMR}\left(\mathrm{CDCl}_{3}\right) \delta: 3.50$ $\left(2 \mathrm{H}, \mathrm{t}, J=7 \mathrm{~Hz},-\mathrm{CH}_{2} \mathrm{OBn}\right), 4.53\left(2 \mathrm{H}, \mathrm{s},-\mathrm{OCH}_{2} \mathrm{Ph}\right), 7.40(5 \mathrm{H}, \mathrm{s}, \mathrm{Ph}) .{ }^{19} \mathrm{~F}-\mathrm{NMR}\left(\mathrm{CDCl}_{3}\right) \dot{\delta}:-17.7(3 \mathrm{~F}, \mathrm{t}, J=9.4 \mathrm{~Hz})$, 
$-51.8(2 \mathrm{~F}, \mathrm{~m}),-64.5(2 \mathrm{~F}, \mathrm{~m}) . \mathrm{MS} m / z: 458\left(\mathrm{M}^{+}\right), 367,348,321,91$. High-resolution MS Calcd for $\mathrm{C}_{23} \mathrm{H}_{33} \mathrm{~F}_{7} \mathrm{O}$ : 458.2416. Found: 458.2390 .

14,14,15,15,16,16,16-Heptafluorohexadecan-1-ol (8b)-Hydrogenolysis of the benzyl ether $7 \mathrm{~b}(433 \mathrm{mg}$, $0.95 \mathrm{mmol})$ on $5 \% \mathrm{Pd}-\mathrm{C}$ in $\mathrm{EtOH}(2 \mathrm{ml})$ and acetic acid $(0.5 \mathrm{ml})$ under atmospheric pressure, followed by recrystallization from $n$-hexane gave $8 \mathbf{b}(281 \mathrm{mg}, 80 \%)$. 8b: mp $49.5-51{ }^{\circ} \mathrm{C} .{ }^{1} \mathrm{H}-\mathrm{NMR}\left(\mathrm{CDCl}_{3}\right) \cdot \delta: 1.20-1.85(23 \mathrm{H}$, $\mathrm{m}), 1.92-2.30\left(2 \mathrm{H}, \mathrm{m}, \mathrm{C}_{3} \mathrm{~F}_{7} \mathrm{CH}_{2}-\right), 3.67\left(2 \mathrm{H}, \mathrm{t}, J=7 \mathrm{~Hz},-\mathrm{CH}_{2} \mathrm{OH}\right) .{ }^{19} \mathrm{~F}-\mathrm{NMR}\left(\mathrm{CDCl}_{3}\right) \delta:-17.8(3 \mathrm{~F}, \mathrm{t}, J=9.4 \mathrm{~Hz})$, $-51.8(2 \mathrm{~F}, \mathrm{~m}),-64.5(2 \mathrm{~F}, \mathrm{~m})$. MS $m / z: 350\left(\mathrm{M}^{+}-18\right), 322,294,280$. Anal. Calcd for $\mathrm{C}_{16} \mathrm{H}_{27} \mathrm{~F}_{7} \mathrm{O}: \mathrm{C}, 52.17 ; \mathrm{H}, 7.39$; F, 36.10. Found: C, 52.23; H, 7.16; F, 36.15.

1-Benzyloxy-16,16,17,17,18,18,18-heptafluorooctadecane (7c)- $-85 \%$ yield. ${ }^{1} \mathrm{H}-\mathrm{NMR}\left(\mathrm{CDCl}_{3}\right) \delta: 3.50(2 \mathrm{H}, \mathrm{t}$, $\left.J=6 \mathrm{~Hz},-\mathrm{CH}_{2} \mathrm{OBn}\right), 4.53\left(2 \mathrm{H}, \mathrm{s},-\mathrm{OCH}_{2} \mathrm{Ph}\right), 7.43(5 \mathrm{H}, \mathrm{s}, \mathrm{Ph}) .{ }^{19} \mathrm{~F}-\mathrm{NMR}\left(\mathrm{CDCl}_{3}\right) \delta:-17.9(3 \mathrm{~F}, \mathrm{t}, J=9.4 \mathrm{~Hz}),-52.3$ $(2 \mathrm{~F}, \mathrm{~m}),-65.0(2 \mathrm{~F}, \mathrm{~m})$. MS $m / z: 486\left(\mathrm{M}^{+}\right), 395,376,91$.

16,16,17,17,18,18,18-Heptafluorooctadecan-1-ol (8c)-87\% yield; mp 55-57 ${ }^{\circ} \mathrm{C}$ ( $n$-hexane). ${ }^{1} \mathrm{H}-\mathrm{NMR}\left(\mathrm{CDCl}_{3}\right)$ $\delta: 1.20-1.85(27 \mathrm{H}, \mathrm{m}), 1.92-2.30\left(2 \mathrm{H}, \mathrm{m}, \mathrm{C}_{3} \mathrm{~F}_{7} \mathrm{CH}_{2}-\right), 3.67\left(2 \mathrm{H}, \mathrm{t}, J=7 \mathrm{~Hz},-\mathrm{CH}_{2} \mathrm{OH}\right) .{ }^{19} \mathrm{~F}-\mathrm{NMR}\left(\mathrm{CDCl}_{3}\right) \delta$ : $-17.6(3 \mathrm{~F}, \mathrm{t}, J=9.4 \mathrm{~Hz}),-52.3(2 \mathrm{~F}, \mathrm{~m}),-65.0(2 \mathrm{~F}, \mathrm{~m}) . \mathrm{MS} m / z: 378\left(\mathrm{M}^{+}-18\right), 350,322$. Anal. Calcd for $\mathrm{C}_{18} \mathrm{H}_{31} \mathrm{~F}_{7} \mathrm{O}: \mathrm{C}, 54.54 ; \mathrm{H}, 7.88 ; \mathrm{F}, 33.55$. Found: $\mathrm{C}, 54.33 ; \mathrm{H}, 7.90 ; \mathrm{F}, 33.72$.

Pentadecafluoroheptadecan-1-ol (4c) A mixture of perfluoroheptyl iodide $(7.18 \mathrm{~g}, 14.5 \mathrm{mmol})$, 9-decenyl acetate $(2.41 \mathrm{~g}, 12 \mathrm{mmol})$ and $\mathrm{Fe}_{3}(\mathrm{CO})_{12}(50 \mathrm{mg})$ was heated at $60^{\circ} \mathrm{C}$ for $1 \mathrm{~h}$, and then at $80^{\circ} \mathrm{C}$ for $1 \mathrm{~h}$. The reaction mixture was dissolved in a mixture of acetic acid $(30 \mathrm{ml})$ and ether $(30 \mathrm{ml})$, and treated with $\mathrm{Zn}$ powder at room temperature for $3 \mathrm{~h}$ to give the crude acetate $(4.82 \mathrm{~g})$, which was saponified with $2.5 \%$ methanolic $\mathrm{KOH}(35 \mathrm{ml})$ at room temperature for $5 \mathrm{~h}$. After extractive work-up (ether for extraction) and evaporation of the solvent, the residue -was purified by sublimation $\left(57-59^{\circ} \mathrm{C} / 3 \mathrm{mmHg}\right)$ to give $4 \mathrm{c}\left(2.751 \mathrm{~g}, 43 \%\right.$ overall yield). $4 \mathrm{c}: \mathrm{mp} 61-63^{\circ} \mathrm{C}$. ${ }^{1} \mathrm{H}-\mathrm{NMR}$ $\left(\mathrm{CDCl}_{3}\right) \delta: 1.23-1.90(16 \mathrm{H}, \mathrm{m}), 1.42(1 \mathrm{H}, \mathrm{s},-\mathrm{OH}), 1.92-2.50\left(2 \mathrm{H}, \mathrm{m}, \mathrm{C}_{7} \mathrm{~F}_{15} \mathrm{CH}_{2}-\right), 3.68\left(2 \mathrm{H}, \mathrm{t}, J=6 \mathrm{~Hz},-\mathrm{CH}_{2} \mathrm{OH}\right)$. ${ }^{19} \mathrm{~F}-\mathrm{NMR}\left(\mathrm{CDCl}_{3}\right) \delta:-17.3(3 \mathrm{~F}, \mathrm{t}, J=9.4 \mathrm{~Hz}),-50.2(2 \mathrm{~F}, \mathrm{~m}),-58.0(8 \mathrm{~F}, \mathrm{~m}),-62.0(2 \mathrm{~F}, \mathrm{~m})$. Anal. Calcd for $\mathrm{C}_{17} \mathrm{H}_{21} \mathrm{~F}_{15} \mathrm{O}: \mathrm{C}, 38.80 ; \mathrm{H}, 4.02 ; \mathrm{F}, 54.14$. Found: $\mathrm{C}, 38.76 ; \mathrm{H}, 4.00 ; \mathrm{F}, 53.93$.

Methanesulfonylated Fluoroalcohols (11a-d)—The alcohols 8a, 8b, 8c and $4 \mathbf{c}(0.5 \mathrm{mmol})$ were each dissolved in dry pyridine $(5 \mathrm{ml})$ and methanesulfonyl chloride $(\mathrm{MsCl})(63 \mathrm{mg}, 0.55 \mathrm{mmol})$ was added at $0{ }^{\circ} \mathrm{C}$. Each mixture was stirred at room temperature for $1 \mathrm{~h}$. The reaction mixture was poured into ice-water, and extracted with ether. The ethereal solution was washed with dil. $\mathrm{HCl}$ and brine, then dried on $\mathrm{MgSO}_{4}$, and concentrated to afford 11a, 11b, 11c or 11d, respectively as white crystals.

11a: $(m=15, n=0)$ yield (Y.) $83 \%$, mp $45-46^{\circ} \mathrm{C} .{ }^{1} \mathrm{H}-\mathrm{NMR}\left(\mathrm{CDCl}_{3}\right) \delta: 1.2-1.48(\mathrm{~m}, 24 \mathrm{H}), 1.90-2.24(\mathrm{~m}, 2 \mathrm{H})$, $1.84-2.36(\mathrm{~m}, 2 \mathrm{H}), 3.00(\mathrm{~s}, 3 \mathrm{H}), 4.20(\mathrm{t}, J=7 \mathrm{~Hz}, 2 \mathrm{H})$. IR (KBr): $3070,2950,2880,1480,1355,1192,1140 \mathrm{~cm}^{-1}$. MS $m / z: 374\left(\mathrm{M}^{+}\right)$.

11b: $(m=13, n=2)$ Y. $87 \%$, mp 39-42 ${ }^{\circ} \mathrm{C} .{ }^{1} \mathrm{H}-\mathrm{NMR}\left(\mathrm{CDCl}_{3}\right) \delta: 1.10-1.90(\mathrm{~m}, 22 \mathrm{H}), 1.90-2.24(\mathrm{~m}, 2 \mathrm{H}), 2.98$ (s, 3H), 4.20 (t, $J=7 \mathrm{~Hz}, 2 \mathrm{H})$. IR (KBr): 2940, 2860, 1473, 1356, 1172, 1126, 1115, $1050 \mathrm{~cm}^{-1}$. MS $m / z: 446\left(\mathrm{M}^{+}\right)$.

11c: $(m=15, n=2)$ Y. $100 \%$, mp 49-51 ${ }^{\circ} \mathrm{C} .{ }^{1} \mathrm{H}-\mathrm{NMR}\left(\mathrm{CDCl}_{3}\right) \delta: 1.08-1.27(\mathrm{~m}, 24 \mathrm{H}), 1.27-1: 87(\mathrm{~m}, 2 \mathrm{H})$, $1.87-2.20(\mathrm{~m}, 2 \mathrm{H}), 2.97(\mathrm{~s}, 3 \mathrm{H}), 4.20(\mathrm{t}, J=7 \mathrm{~Hz}, 2 \mathrm{H})$. IR $(\mathrm{KBr}): 2940,2860,1473,1356,1172,1126,1115$, $1050 \mathrm{~cm}^{-1}$. MS $m / z: 474\left(\mathrm{M}^{+}\right)$.

11d: $(m=10, n=6)$ Y. $87 \%$, mp 59.5-62.5 ${ }^{\circ} \mathrm{C} .{ }^{1} \mathrm{H}-\mathrm{NMR}\left(\mathrm{CDCl}_{3}\right) \delta: 1.13-1.44(\mathrm{~m}, 14 \mathrm{H}), 1.44-1.88(\mathrm{~m}, 2 \mathrm{H})$, $1.89-2.25(\mathrm{~m}, 2 \mathrm{H}), 2.97(\mathrm{~s}, 3 \mathrm{H}), 4.20(\mathrm{t}, J=7 \mathrm{~Hz}, 2 \mathrm{H})$. IR (KBr): 2940, 2860, 1472, 1357, 1170, 1126, 1116, 1052, $1006 \mathrm{~cm}^{-1}$. MS $m / z: 604\left(\mathrm{M}^{+}\right)$.

1-O-Fluoroalkyl-2-O-benzyl-3,4-O-isopropylidene-D-threitols $(12 \mathrm{a}-\mathrm{d})-\mathrm{A} 22 \%$ potassium hydride oil dispersion $(0.27 \mathrm{ml}, 1.5 \mathrm{mmol})$ was slowly added to a solution of $2-O$-benzyl-3,4- $O$-isopropylidene-D-threitol ${ }^{5)}(175 \mathrm{mg}$, $0.7 \mathrm{mmol})$ in benzene $(10 \mathrm{ml})$ at room temperature. The reaction mixture was stirred for an additional $30 \mathrm{~min}$, and then $11(0.46 \mathrm{mmol})$ in benzene $(10 \mathrm{ml})$ was added. The reaction mixture was stirred for $10 \mathrm{~h}$ at room temperature, cooled to $0{ }^{\circ} \mathrm{C}$, diluted with hexane $(10 \mathrm{ml})$, and quenched by successive addition of EtOH $(1 \mathrm{ml})$ and water $(5 \mathrm{ml})$. The organic layer was separated, and the aqueous layer was washed with AcOEt. The combined organic layer was dried on $\mathrm{MgSO}_{4}$, concentrated and purified by silica gel column chromatography (benzene) to afford 12 as a colorless oil.

12a: $(m=15, n=0) \mathrm{Y} .66 \% \cdot{ }^{1} \mathrm{H}-\mathrm{NMR}\left(\mathrm{CDCl}_{3}\right) \delta: 1.17-1.34(\mathrm{~m}, 24 \mathrm{H}), 1.39(\mathrm{~s}, 3 \mathrm{H}), 1.43(\mathrm{~s}, 3 \mathrm{H}), 1.57-1.87(\mathrm{~m}$, $2 \mathrm{H}), 1.87-2.31(\mathrm{~m}, 2 \mathrm{H}), 3.41(\mathrm{t}, J=7 \mathrm{~Hz}, 2 \mathrm{H}), 3.51-4.40(\mathrm{~m}, 6 \mathrm{H}), 4.78(\mathrm{~s}, 2 \mathrm{H}), 7.27-7.47(\mathrm{~m}, 5 \mathrm{H})$. IR (neat): 3450 , $2920,2860,1455,1370,1220,1160 \mathrm{~cm}^{-1}$. MS $m / z: 530\left(\mathrm{M}^{+}-15\right)$.

12b: $(m=13, n=2)$ Y. $75 \% \cdot{ }^{1} \mathrm{H}-\mathrm{NMR}\left(\mathrm{CDCl}_{3}\right) \delta: 1.16-1.72(\mathrm{~m}, 22 \mathrm{H}), 1.36(\mathrm{~s}, 3 \mathrm{H}), 1.42(\mathrm{~s}, 3 \mathrm{H}), 1.90-2.40(\mathrm{~m}$, $2 \mathrm{H}), 3.38(\mathrm{t}, J=12 \mathrm{~Hz}, 2 \mathrm{H}), 3.47-4.40(\mathrm{~m}, 6 \mathrm{H}), 4.71(\mathrm{~s}, 2 \mathrm{H}), 7.16-7.48(\mathrm{~m}, 5 \mathrm{H})$. IR (neat): 3450, 2920, 2860, 1445, $1370,1150 \mathrm{~cm}^{-1}$. MS $m / z: 602\left(\mathrm{M}^{+}\right), 587\left(\mathrm{M}^{+}-15\right)$.

12c: $(m=15, n=2)$ Y. $89 \%{ }^{1} \mathrm{H}-\mathrm{NMR}\left(\mathrm{CDCl}_{3}\right) \delta: 1: 16-1.72(\mathrm{~m}, 26 \mathrm{H}), 1.36(\mathrm{~s}, 3 \mathrm{H}), 1.40(\mathrm{~s}, 3 \mathrm{H}), 1.89-2.28(\mathrm{~m}$, $2 \mathrm{H}), 3.41(\mathrm{t}, J=7 \mathrm{~Hz}, 2 \mathrm{H}), 3.51-4.40(\mathrm{~m}, 6 \mathrm{H}), 4.72(\mathrm{~s}, 2 \mathrm{H}), 7.28(\mathrm{~m}, 5 \mathrm{H})$. IR (neat): 3450, 2920, 2860, 1455, 1370, $1160 \mathrm{~cm}^{-1}$. MS $m / z: 630\left(\mathrm{M}^{+}\right), 615\left(\mathrm{M}^{+}-15\right)$.

12d: $(m=10, n=6)$ Y. $66 \% .{ }^{1} \mathrm{H}-\mathrm{NMR}\left(\mathrm{CDCl}_{3}\right) \delta: 1.16-1.48(\mathrm{~m}, 14 \mathrm{H}), 1.34(\mathrm{~s}, 3 \mathrm{H}), 1.43(\mathrm{~s}, 3 \mathrm{H}), 1.49-1.88(\mathrm{~m}$ $2 \mathrm{H}), 1.89-2.25(\mathrm{~m}, 2 \mathrm{H}), 3.40(\mathrm{t}, J=7 \mathrm{~Hz}, 2 \mathrm{H}), 3.44-4.10(\mathrm{~m}, 5 \mathrm{H}), 4.10-4.30(\mathrm{~m}, 1 \mathrm{H}), 4.75(\mathrm{~s}, 2 \mathrm{H}), 7.22-7.48(\mathrm{~s}$, 5H). IR (neat): $3450,2920,2840,1455,1370,1160 \mathrm{~cm}^{-1}$. MS $m / z: 760\left(\mathrm{M}^{+}\right), 745\left(\mathrm{M}^{+}-15\right)$.

1-O-Fluoroalkyl-2-O-benzyl-D-threitols $(13 \mathrm{a}-\mathrm{d})$-The $O$-alkylated derivative $12(0.4 \mathrm{mmol})$ was stirred in 
THF $(15 \mathrm{ml})$ and $2 \mathrm{~N} \mathrm{HCl}(5 \mathrm{ml})$ at room temperature for $2 \mathrm{~h}$, then the solution was concentrated to a small volume (ca. $5 \mathrm{ml})$ under reduced pressure. After water $(10 \mathrm{ml})$ had been added, the mixture was extracted with ethyl acetate $(20 \mathrm{ml} \times 3)$. Evaporation of the solvent gave a residue, which was chromatographed on silica gel to give $\mathbf{1 3}$ as white crystals.

13a: Y. $73 \%$, mp 36-38 C. ${ }^{1} \mathrm{H}-\mathrm{NMR}\left(\mathrm{CDCl}_{3}\right) \delta: 1.17-1.48(\mathrm{~m}, 24 \mathrm{H}), 1.48-1.76(\mathrm{~m}, 2 \mathrm{H}), 1.87-2.31(\mathrm{~m}, 4 \mathrm{H})$, $3.41(\mathrm{t}, J=7 \mathrm{~Hz}, 2 \mathrm{H}), 3.60-3.88(\mathrm{~m}, 6 \mathrm{H}), 4.38(\mathrm{~d}, J=12 \mathrm{~Hz}, 1 \mathrm{H}), 4.78(\mathrm{~d}, J=12 \mathrm{~Hz}, 1 \mathrm{H}), 7.27-7.37(\mathrm{~m}, 5 \mathrm{H}) . \mathrm{IR}$ (KBr): 3300, 2920, 2860, 1460, 1210,1150 $\mathrm{cm}^{-1}$.

13b: Y. $77 \%$, mp 32-34 C. ${ }^{1} \mathrm{H}-\mathrm{NMR}\left(\mathrm{CDCl}_{3}\right) \delta: 1.15-1.85(\mathrm{~m}, 22 \mathrm{H}), 1.92-2.20(\mathrm{~m}, 2 \mathrm{H}), 2.85(\mathrm{brs}, 2 \mathrm{H}), 3.42$ $(\mathrm{t}, J=7 \mathrm{~Hz}, 2 \mathrm{H}), 3.55-3.90(\mathrm{~m}, 6 \mathrm{H}), 4.53(\mathrm{~d}, J=12 \mathrm{~Hz}, 1 \mathrm{H}), 4.73(\mathrm{~d}, J=12 \mathrm{~Hz}, 1 \mathrm{H}), 7.17-7.52(\mathrm{~m}, 5 \mathrm{H}) . \mathrm{IR}(\mathrm{KBr})$ : $3350,2940,2880,1240,1210,1160 \mathrm{~cm}^{-1}$.

13c: Y. $76 \%$ mp $41-44{ }^{\circ} \mathrm{C} .{ }^{1} \mathrm{H}-\mathrm{NMR}\left(\mathrm{CDCl}_{3}\right) \delta: 1.15-1.72(\mathrm{~m}, 26 \mathrm{H}), 1.91-2.20(\mathrm{~m}, 4 \mathrm{H}), 3.42(\mathrm{t}, J=7 \mathrm{~Hz}$, $2 \mathrm{H}), 3.53-3.87(\mathrm{~m}, 6 \mathrm{H}), 4.55(\mathrm{~d}, J=12 \mathrm{~Hz}, 1 \mathrm{H}), 4.75(\mathrm{~d}, J=12 \mathrm{~Hz}, 1 \mathrm{H}), 7.29(\mathrm{~s}, 5 \mathrm{H})$. IR (KBr): 3450, 2920, 2840, $1470,1355,1210,1150 \mathrm{~cm}^{-1}$. CI-MS $m / z: 591\left(\mathrm{M}^{+}+1\right)$.

13d: Y. $88 \%$, mp $51-54{ }^{\circ} \mathrm{C} .{ }^{1} \mathrm{H}-\mathrm{NMR}\left(\mathrm{CDCl}_{3}\right) \delta: 1.16-1.48(\mathrm{~m}, 14 \mathrm{H}), 1.49-1.88(\mathrm{~m}, 2 \mathrm{H}), 1.89-2.25(\mathrm{~m}, 2 \mathrm{H})$, 2.79 (br s, 2H), $3.44(\mathrm{t}, J=7 \mathrm{~Hz}, 2 \mathrm{H}), 3.55-3.88(\mathrm{~m}, 6 \mathrm{H}), 4.56(\mathrm{~d}, J=12 \mathrm{~Hz}, 1 \mathrm{H}), 4.76(\mathrm{~d}, J=12 \mathrm{~Hz}, 1 \mathrm{H}), 7.32(\mathrm{~s}, 5 \mathrm{H})$. IR (KBr): $3350,2940,2880,1240,1210,1150 \mathrm{~cm}^{-1}$.

1-O-Fluoroalkyl-2-O-benzyl-sn-glycerols (14a-d)-Under vigorous stirring $13(0.3 \mathrm{mmol})$ in benzene $(10 \mathrm{ml})$ was added to a solution of $\mathrm{Pb}(\mathrm{OAc})_{4}(200 \mathrm{mg}, 0.45 \mathrm{mmol})$ in benzene $(20 \mathrm{ml})$ over a $2 \mathrm{~h}$ period at room temperature. After being stirred for an additional hour, the reaction mixture was cooled to $0{ }^{\circ} \mathrm{C}$ and saturated aqueous sodium bicarbonate was added. The resulting insoluble material was filtered through a celite layer and washed with benzene. The organic layer was separated, dried over $\mathrm{Na}_{2} \mathrm{SO}_{4}$, and concentrated to afford the corresponding crude aldehyde in quantitative yield as a colorless oil. This was used without further purification for the next step. $\mathrm{NaBH}_{4}(113 \mathrm{mg}$, $3 \mathrm{mmol})$ was slowly added to a solution of the crude aldehyde $(0.3 \mathrm{mmol})$ in methanol $(10 \mathrm{ml})$ at room temperature. The reaction mixture was allowed to stand at room temperature overnight, cooled to $0{ }^{\circ} \mathrm{C}$, acidified with dil. hydrochloric acid, and concentrated to dryness under reduced pressure. The product was extracted with ethyl acetate $(20 \mathrm{ml} \times 3)$, and the organic layer was dried over $\mathrm{Na}_{2} \mathrm{SO}_{4}$. After the removal of the solvent, the residue was chromatographed on silica gel to afford 14 as a colorless oil $(\mathbf{1 4 a}, \mathbf{b}, \mathbf{d})$ or white crystals (14c).

14a: Y. $81 \%$, oil. ${ }^{1} \mathrm{H}-\mathrm{NMR}\left(\mathrm{CDCl}_{3}\right) \delta: 1.17-1.48(\mathrm{~m}, 24 \mathrm{H}), 1.49-1.76(\mathrm{~m}, 2 \mathrm{H}), 1.87-2.31(\mathrm{~m}, 3 \mathrm{H}), 3.41(\mathrm{t}, J=$ $7 \mathrm{~Hz}, 2 \mathrm{H}), 3.44-3.80(\mathrm{~m}, 5 \mathrm{H}), 4.62(\mathrm{~d}, J=12 \mathrm{~Hz}, 1 \mathrm{H}), 4.64(\mathrm{~d}, J=12 \mathrm{~Hz}, 1 \mathrm{H}), 7.28(\mathrm{~s}, 5 \mathrm{H})$. IR (neat): 3400, 2910 , $2840,1460,1450,1230,1135 \mathrm{~cm}^{-1} \cdot[\alpha]_{\mathrm{D}}^{20}-5.98^{\circ}\left(c=0.57, \mathrm{CHCl}_{3}\right) . \mathrm{MS} m / z: 460\left(\mathrm{M}^{+}\right)$.

14b: Y. $72 \%$, oil. ${ }^{1} \mathrm{H}-\mathrm{NMR}\left(\mathrm{CDCl}_{3}\right) \delta: 1.15-1.85(\mathrm{~m}, 22 \mathrm{H}), 1.86-2.37(\mathrm{~m}, 3 \mathrm{H}), 3.43(\mathrm{t}, J=7 \mathrm{~Hz}, 2 \mathrm{H}), 3.53-$ $3.97(\mathrm{~m}, 5 \mathrm{H}), 4.67(\mathrm{~s}, 2 \mathrm{H}), 7.21-7.51(\mathrm{~m}, 5 \mathrm{H})$. IR (neat): $3400,2920,2840,1460,1450,1230,1140 \mathrm{~cm}^{-1} \cdot[\alpha]_{\mathrm{D}}^{20}-12.4$ $\left(c=0.54, \mathrm{CHCl}_{3}\right)$. MS $m / z: 532\left(\mathrm{M}^{+}\right)$.

14c: Y. $72 \%$, white crystals, mp 36-38 ${ }^{\circ} \mathrm{C} .{ }^{1} \mathrm{H}-\mathrm{NMR}\left(\mathrm{CDCl}_{3}\right) \delta: 1.16-1.72(\mathrm{~m}, 26 \mathrm{H}), 1.91-2.20(\mathrm{~m}, 2 \mathrm{H}), 3.41$ $(\mathrm{t}, J=7 \mathrm{~Hz}, 2 \mathrm{H}), 3.51-3.95(\mathrm{~m}, 5 \mathrm{H}), 4.64(\mathrm{~s}, 2 \mathrm{H}), 7.21-7.47(\mathrm{~m}, 5 \mathrm{H}) . \mathrm{IR}(\mathrm{KBr}): 3450,2920,2840,1470,1355,1205$, $1140 \mathrm{~cm}^{-1} \cdot[\alpha]_{\mathrm{D}}^{20}-9.28^{\circ}\left(c=1.12, \mathrm{CHCl}_{3}\right) . \mathrm{MS} m / z: 560\left(\mathrm{M}^{+}\right)$.

14d: Y. $73 \%$, oil. ${ }^{1} \mathrm{H}-\mathrm{NMR}\left(\mathrm{CDCl}_{3}\right) \delta: 1.16-1.48(\mathrm{~m}, 14 \mathrm{H}), 1.49-1.88(\mathrm{~m}, 2 \mathrm{H}), 1.89-2.25(\mathrm{~m}, 2 \mathrm{H}), 3.40(\mathrm{t}, J=$ $7 \mathrm{~Hz}, 2 \mathrm{H}), 3.44-3.80(\mathrm{~m}, 5 \mathrm{H}), 4.62(\mathrm{~d}, J=12 \mathrm{~Hz}, 1 \mathrm{H}), 4.64(\mathrm{~d}, J=12 \mathrm{~Hz}, 1 \mathrm{H}), 7.28(\mathrm{~s}, 5 \mathrm{H})$. IR (neat): 3350, 2920 , $2840,1465,1452,1320,1240,1210,1140 \mathrm{~cm}^{-1} \cdot[\alpha]_{\mathrm{D}}^{20}-6.20^{\circ}\left(c=1.37, \mathrm{CHCl}_{3}\right) . \mathrm{MS} m / z: 704\left(\mathrm{M}^{+}\right)$.

1-O-Fluoroalkyl-2-O-benzyl-sn-glycero-3-phosphocholines (15a-d) A mixture of 15 (0.19 mmol), 2-bromoethyl phosphoryl dichloride $(69 \mathrm{mg}, 0.29 \mathrm{mmol})$ and triethylamine $(39 \mathrm{mg}, 0.38 \mathrm{mmol})$ in dry ether $(10 \mathrm{ml})$ was stirred under reflux for $12 \mathrm{~h}$. Then water $(0.2 \mathrm{ml})$ and triethylamine $(0.1 \mathrm{ml})$ were added, and the reaction mixture was heated under reflux for $1 \mathrm{~h}$. After cooling, $1 \mathrm{~N} \mathrm{HCl}(5 \mathrm{ml})$ was added and the mixture was extracted with chloroform $(20 \mathrm{ml} \times 3)$. The organic layer was dried on $\mathrm{MgSO}_{4}$ and concentrated to afford crude 2-bromoethyl 2-O-benzyl-L-1-Ofluoroalkyl-glyceryl hydrogen phosphate. The product was used without further purification in the next step. The phosphate was heated with trimethylamine $(1 \mathrm{ml})$ and methanol $(2 \mathrm{ml})$ to $55^{\circ} \mathrm{C}$ in a sealed tube for $12 \mathrm{~h}$. After removal of the solvent, $\mathrm{AgOAc}(80 \mathrm{mg}, 0.48 \mathrm{mmol})$ in $90 \%$ aqueous methanol $(20 \mathrm{ml})$ was added, and the reaction mixture was stirred for $2 \mathrm{~h}$. The resulting precipitate was removed by filtration, and the filtrate was concentrated to give crude 15. The product was purified by silica gel column chromatography to afford $\mathbf{1 5}$ as a white powder.

15a: Y. $52 \%$ mp ca. $190{ }^{\circ} \mathrm{C} .{ }^{1} \mathrm{H}-\mathrm{NMR}\left(\mathrm{CDCl}_{3}-\mathrm{CD}_{3} \mathrm{OD}, 3: 1\right) \delta: 1.17-1.48(\mathrm{~m}, 24 \mathrm{H}), 1.49-1.76(\mathrm{~m}, 2 \mathrm{H})$, $1.87-2.31(\mathrm{~m}, 2 \mathrm{H}), 3.09(\mathrm{~s}, 9 \mathrm{H}), 3.28-3.64(\mathrm{~m}, 9 \mathrm{H}), 3.88-4.10(\mathrm{~m}, 2 \mathrm{H}), 4.69(\mathrm{~s}, 2 \mathrm{H}), 7.18-7.40(\mathrm{~m}, 5 \mathrm{H}) . \mathrm{IR}(\mathrm{KBr})$ : $3390,3070,3040,2930,2860,1660,1460,1320,1220,1150 \mathrm{~cm}^{-1} \cdot[\alpha]_{\mathrm{D}}^{20}+1.20^{\circ}\left(c=0.40, \mathrm{CHCl}_{3}-\mathrm{MeOH}_{1} 1: 1\right)$.

15b: Y. $86 \%, \mathrm{mp} 135-142{ }^{\circ} \mathrm{C} .{ }^{1} \mathrm{H}-\mathrm{NMR}\left(\mathrm{CDCl}_{3}-\mathrm{CD}_{3} \mathrm{OD}, 3: 1\right) \delta: 1.10-1.85(\mathrm{~m}, 22 \mathrm{H}), 1.90-2.33(\mathrm{~m}, 2 \mathrm{H})$, $3.26(\mathrm{~s}, 9 \mathrm{H}), 3.41(\mathrm{t}, J=7 \mathrm{~Hz}, 2 \mathrm{H}), 3.30-3.70(\mathrm{~m}, 4 \mathrm{H}), 3.70-4.37(\mathrm{~m}, 5 \mathrm{H}), 4.70(\mathrm{~s}, 2 \mathrm{H}), 7.18-7.50(\mathrm{~m}, 5 \mathrm{H}) . \mathrm{IR}$ (KBr): $3420,2940,2850,1465,1230,1085 \mathrm{~cm}^{-1} \cdot[\alpha]_{\mathrm{D}}^{20}+3.81^{\circ}\left(c=0.42, \mathrm{CHCl}_{3}-\mathrm{MeOH}, 1: 1\right)$.

15c: Y. $68 \%$, mp 229-233 ${ }^{\circ} \mathrm{C}$ (dec.). ${ }^{1} \mathrm{H}-\mathrm{NMR}\left(\mathrm{CDCl}_{3}-\mathrm{CD}_{3} \mathrm{OD}, 3: 1\right) \delta: 1.10-1.73(\mathrm{~m}, 26 \mathrm{H}), 1.91-2.33(\mathrm{~m}$, $2 \mathrm{H}), 3.11(\mathrm{~s}, 9 \mathrm{H}), 3.41(\mathrm{t}, J=7 \mathrm{~Hz}, 2 \mathrm{H}), 3.30-3.70(\mathrm{~m}, 4 \mathrm{H}), 3.70-4.37(\mathrm{~m}, 5 \mathrm{H}), 4.70(\mathrm{~s}, 2 \mathrm{H}), 7.18-7.50(\mathrm{~m}, 5 \mathrm{H}) . \mathrm{IR}$ (KBr): $3420,2940,2850,1465,1230,1085 \mathrm{~cm}^{-1} \cdot[\alpha]_{\mathrm{D}}^{20}+2.61^{\circ}\left(c=0.94, \mathrm{CHCl}_{3}-\mathrm{MeOH}_{1} 1: 1\right)$.

15d: Y. $60 \%$, mp $223-226{ }^{\circ} \mathrm{C} .{ }^{1} \mathrm{H}-\mathrm{NMR}\left(\mathrm{CDCl}_{3}-\mathrm{CD}_{3} \mathrm{OD}, 3: 1\right) \delta: 1.16-1.83(\mathrm{~m}, 16 \mathrm{H}), 1.90-2.37(\mathrm{~m}, 2 \mathrm{H})$, $3.09(\mathrm{~s}, 9 \mathrm{H}), 3.28-3.64(\mathrm{~m}, 9 \mathrm{H}), 3.88-4.10(\mathrm{~m}, 2 \mathrm{H}), 4.69(\mathrm{~s}, 2 \mathrm{H}), 7.23(\mathrm{~s}, 5 \mathrm{H}) . \mathrm{IR}(\mathrm{KBr}): 3400,2940,2860,1460$, 
$1230,1085 \mathrm{~cm}^{-1} \cdot[\alpha]_{\mathrm{D}}^{20}+2.11^{\circ}\left(c=1.11, \mathrm{CHCl}_{3}-\mathrm{MeOH}, 1: 1\right)$.

1-O-Fluoroalkyl-sn-glycero-3-phosphocholines $(16 \mathrm{a}-\mathrm{d})$ - A mixture of $15(0.13 \mathrm{mmol})$ and palladium black $(10 \mathrm{mg})$ in ethanol was vigorously stirred under a hydrogen atmosphere $(1 \mathrm{~atm})$ for $6 \mathrm{~h}$ at $50^{\circ} \mathrm{C}$. The catalyst was filtered off, and the filtrate was concentrated. The residual powder was purified on silica gel column chromatography to afford $\mathbf{1 6}$ as a white powder.

16a: Y. $100 \%$, mp $230{ }^{\circ} \mathrm{C}$ (dec.). ${ }^{1} \mathrm{H}-\mathrm{NMR}\left(\mathrm{CDCl}_{3}-\mathrm{CD}_{3} \mathrm{OD}, 3: 1\right) \delta: 1.17-1.48(\mathrm{~m}, 24 \mathrm{H}), 1.49-1.76(\mathrm{~m}, 2 \mathrm{H})$, $1.87-2.31(\mathrm{~m}, 2 \mathrm{H}), 3.21(\mathrm{~s}, 9 \mathrm{H}), 3.30-3.76(\mathrm{~m}, 9 \mathrm{H}), 3.88-4.10(\mathrm{~m}, 2 \mathrm{H}) . \mathrm{IR}(\mathrm{KBr}): 3420,2940,2850,1465,1210$, $1150 \mathrm{~cm}^{-1} \cdot[\alpha]_{\mathrm{D}}^{20}-2.40^{\circ}\left(c=0.35, \mathrm{CHCl}_{3}-\mathrm{MeOH}, 1: 1\right)$.

16b: Y. $85 \%$, mp $196{ }^{\circ} \mathrm{C}$ (dec.). ${ }^{1} \mathrm{H}-\mathrm{NMR}\left(\mathrm{CDCl}_{3}-\mathrm{CD}_{3} \mathrm{OD}, 3: 1\right) \delta: 1.10-1.85(\mathrm{~m}, 22 \mathrm{H}), 1.90-2.35(\mathrm{~m}, 2 \mathrm{H})$, $3.26 .(\mathrm{s}, 9 \mathrm{H}), 3.38-3.76(\mathrm{~m}, 7 \mathrm{H}), 3.77-4.09(\mathrm{~m}, 2 \mathrm{H}), 4.10-4.46(\mathrm{~m}, 2 \mathrm{H})$. IR (KBr): $3400,2940,2860,1472,1360$, $1220,1145 \mathrm{~cm}^{-1} \cdot[\alpha]_{\mathrm{D}}^{20}-2.00^{\circ}\left(c=1.17, \mathrm{CHCl}_{3}-\mathrm{MeOH}, 1: 1\right)$.

16c: Y. $78 \%$, mp 226-234 ${ }^{\circ} \mathrm{C}$ (dec.). ${ }^{1} \mathrm{H}-\mathrm{NMR}\left(\mathrm{CDCl}_{3}-\mathrm{CD}_{3} \mathrm{OD}, 3: 1\right) \delta: 1.10-1.73(\mathrm{~m}, 26 \mathrm{H}), 1.91-2.33(\mathrm{~m}$, $2 \mathrm{H}), 3.25(\mathrm{~s}, 9 \mathrm{H}), 3.33-3.76(\mathrm{~m}, 7 \mathrm{H}), 3.77-3.93(\mathrm{~m}, 2 \mathrm{H}), 3.94-4.40(\mathrm{~m}, 2 \mathrm{H})$. IR (KBr): $3400,2940,2860,1472$, $1360,1220,1140 \mathrm{~cm}^{-1} \cdot[\alpha]_{\mathrm{D}}^{20}-3.61^{\circ}\left(c=0.64, \mathrm{CHCl}_{3}-\mathrm{MeOH}, 1: 1\right)$.

16d: Y. $90 \%$, mp $222-224{ }^{\circ} \mathrm{C}$ (dec.). ${ }^{1} \mathrm{H}-\mathrm{NMR}\left(\mathrm{CDCl}_{3}-\mathrm{CD}_{3} \mathrm{OD}, 3: 1\right) \delta: 1.16-1.83(\mathrm{~m}, 16 \mathrm{H}), 1.90-2.37(\mathrm{~m}$, $2 \mathrm{H}), 3.21(\mathrm{~s}, 9 \mathrm{H}), 3.30-3.94(\mathrm{~m}, 2 \mathrm{H})$. IR $(\mathrm{KBr}): 3420,2940,2850,1465,1210,1140 \mathrm{~cm}^{-1} \cdot[\alpha]_{\mathrm{D}}^{20}-2.55^{\circ}(c=0.55$, $\left.\mathrm{CHCl}_{3}-\mathrm{MeOH}, 1: 1\right)$.

1-O-Fluoroalkyl-2-O-acetyl-sn-glycero-3-phosphocholines $(17 \mathrm{a}-\mathrm{d})-\mathrm{A}$ solution of $16(0.1 \mathrm{mmol})$ in acetic anhydride was mixed with pyridine, and the reaction mixture was heated to $70^{\circ} \mathrm{C}$ for $2 \mathrm{~h}$, then cooled to $50^{\circ} \mathrm{C}$. Pyridine and acetic anhydride were removed under reduced pressure, and the resulting residue was chromatographed on silica gel to afford $\mathbf{1 7}$ as a white powder.

17a: Y. $55 \%$, mp 272-276 C. ${ }^{1} \mathrm{H}-\mathrm{NMR}\left(\mathrm{CDCl}_{3}-\mathrm{CD}_{3} \mathrm{OD}, 3: 1\right) \delta: 1.17-1.48(\mathrm{~m}, 24 \mathrm{H}), 1.49-1.76(\mathrm{~m}, 2 \mathrm{H})$, $1.87-2.31(\mathrm{~m}, 2 \mathrm{H}), 2.12(\mathrm{~s}, 3 \mathrm{H}), 3.21(\mathrm{~s}, 9 \mathrm{H}), 3.45(\mathrm{t}, J=7 \mathrm{~Hz}, 2 \mathrm{H}), 3.59(\mathrm{~d}, J=5 \mathrm{~Hz}, 2 \mathrm{H}), 3.64(\mathrm{~m}, 2 \mathrm{H}), 4.00(\mathrm{~m}, 2 \mathrm{H})$, 5.13 (quintet, $J=5 \mathrm{~Hz}, 1 \mathrm{H})$. IR (KBr): $3420,2920,2850,1732,1627,1465,1374,1235,1140 \mathrm{~cm}^{-1} \cdot[\alpha]_{\mathrm{D}}^{20}-0.46^{\circ}(c=$ $\left.0.11, \mathrm{CHCl}_{3}-\mathrm{MeOH}, 1: 1\right)$. FAB-MS (glycerine) $578\left(\mathrm{M}^{+}+1\right)$.

17b: Y. $38 \%$, mp 272-276 ${ }^{\circ} \mathrm{C}$ (dec.). ${ }^{1} \mathrm{H}-\mathrm{NMR}\left(\mathrm{CDCl}_{3}-\mathrm{CD}_{3} \mathrm{OD}, 3: 1\right) \delta: 1.12-1.54(\mathrm{~m}, 22 \mathrm{H}), 1.55-1.78(\mathrm{~m}$, $2 \mathrm{H}), 2.20(\mathrm{~s}, 3 \mathrm{H}), 3.34(\mathrm{~s}, 9 \mathrm{H}), 3.52-4.00(\mathrm{~m}, 8 \mathrm{H}), 4.20-4.40(\mathrm{~m}, 2 \mathrm{H}), 5.10-5.28(\mathrm{~m}, 1 \mathrm{H})$. IR $\left(\mathrm{CHCl}_{3}\right): 2930,2850$, $1735,1220,1080 \mathrm{~cm}^{-1} \cdot[\alpha]_{\mathrm{D}}^{20}-4.86^{\circ}\left(c=0.10, \mathrm{CHCl}_{3}-\mathrm{CD}_{3} \mathrm{OD}, 1: 1\right)$. FAB-MS (glycerine) $650\left(\mathrm{M}^{+}+1\right)$.

17c: Y. $75 \%$, mp $208-212{ }^{\circ} \mathrm{C}$ (dec.). ${ }^{1} \mathrm{H}-\mathrm{NMR}\left(\mathrm{CDCl}_{3}-\mathrm{CD}_{3} \mathrm{OD}, 3: 1\right) \delta: 1.10-1.73(\mathrm{~m}, 26 \mathrm{H}), 1.91-2.35(\mathrm{~m}$, $2 \mathrm{H}), 2.07(\mathrm{~s}, 3 \mathrm{H}), 3.18(\mathrm{~s}, 9 \mathrm{H}), 3.28-3.70(\mathrm{~m}, 9 \mathrm{H}), 3.88-4.10(\mathrm{~m}, 2 \mathrm{H}), 4.10-4.40(\mathrm{~m}, 2 \mathrm{H}), 5.10-5.25(\mathrm{~m}, 1 \mathrm{H}) .{ }^{19} \mathrm{~F}-$ $\operatorname{NMR}\left(\mathrm{CDCl}_{3}\right):-17.9(\mathrm{t}, J=9.5 \mathrm{~Hz}, 3 \mathrm{~F}),-52.6(\mathrm{~m}, 2 \mathrm{~F}),-65.1(\mathrm{~m}, 2 \mathrm{~F})$. IR $(\mathrm{KBr}): 3350,2940,2860,1735,1465$, $1230,1150 \mathrm{~cm}^{-1} \cdot[\alpha]_{\mathrm{D}}^{20}-1.59^{\circ}\left(c=0.63, \mathrm{CHCl}_{3}-\mathrm{MeOH}, 1: 1\right)$. FAB-MS (glycerine) $678\left(\mathrm{M}^{+}+1\right)$.

17d: Y. $57 \%$, mp 196-199 $\mathrm{C}$ (dec.). ${ }^{1} \mathrm{H}-\mathrm{NMR}\left(\mathrm{CDCl}_{3}-\mathrm{CD}_{3} \mathrm{OD}, 3: 1\right) \delta: 1.16-1.83(\mathrm{~m}, 16 \mathrm{H}), 1.90-2.37(\mathrm{~m}$, $2 \mathrm{H}), 2.90(\mathrm{~s}, 3 \mathrm{H}), 3.20(\mathrm{~s}, 9 \mathrm{H}), 3.45(\mathrm{t}, J=7 \mathrm{~Hz}, 2 \mathrm{H}), 3.53-3.70(\mathrm{~m}, 2 \mathrm{H}), 3.84-4.06(\mathrm{~m}, 2 \mathrm{H}), 4.06-4.40(\mathrm{~m}, 2 \mathrm{H}), 5.12$ (quintet, $J=5 \mathrm{~Hz}, 1 \mathrm{H}) .{ }^{19} \mathrm{~F}-\mathrm{NMR}\left(\mathrm{CDCl}_{3}\right):-18.0(\mathrm{t}, J=9.7 \mathrm{~Hz}, 3 \mathrm{~F}),-51.2(\mathrm{~m}, 2 \mathrm{~F}),-57.7--60.0(\mathrm{~m}, 8 \mathrm{~F}),-63.0$ $(\mathrm{m}, 2 \mathrm{~F})$. IR (KBr): $3340,2940,2860,1735,1465,1230,1150 \mathrm{~cm}^{-1} \cdot[\alpha]_{\mathrm{D}}^{20}-4.75^{\circ}\left(c=0.33, \mathrm{CHCl}_{3}-\mathrm{MeOH}, 1: 1\right)$. FABMS (glycerine) $808\left(\mathrm{M}^{+}+1\right)$.

Acknowledgments We wish to express our thanks to Professor Y. Kasuya and K. Shigenobu for measurement of the hypotensive effect of the synthetic analogues and for helpful discussions.

\section{References and Notes}

1) Studies on Organic Fluorine Compounds. Part LI. Part L, Y. Kobayashi, M. Nakazawa, I. Kumadaki, T. Taguchi, E. Ohshima, N. Ikekawa, Y. Tanaka, and H. F. DeLuca, Chem. Pharm. Bull., 34, 1568 (1986).

2) C. A. Demopoulus, R. N. Pinckard, and D. J. Hanahan, J. Biol. Chem., 254, 9355 (1979).

3) J. Benveniste, M. Tence, P. Varenne, J. Bidault, and J. Polonsky, C. R. Acad. Sci., 289D, 1037 (1979).

4) R. N. Pinckard, L. M. McManus, and D. J. Hanahan, "Advanced Inflammation Research," Vol. 4, ed. by G. Weissman, Raven Press, New York, 1982, p. 147.

5) M. Ohno, K. Fujita, H. Nakai, S. Kobayashi, K. Inoue, and S. Nojima, Chem. Pharm. Bull., 33, 572 (1985).

6) R. Filler, "Organofluorine Chemicals and Their Industrial Applications," ed. by R. E. Banks, Ellis Horwood, Chichester, 1979, p. 123.

7) a) T. Fujita, J. Iwasa, and C. Hansch, J. Am. Chem. Soc., 8o, 5175 (1964); b) C. Hansch and J. Fukunaga, Chemtech., 7, 120 (1977).

8) T. Fuchikami and I. Ojima, Tetrahedron Lett., 25, 303 (1984).

9) M. Tamura and J. Kochi, Synthesis, 1971, 303; G. Fouquest and M. Schlosser, Angew. Chem. Int. Ed. Engl., 13, 82 (1974).

10) H. Hayashi, I. Kudo, K. Inoue, K. Onozaki, S. Tsushima, H. Nomura, and S. Nojima, J. Biochem. (Tokyo), 97, 1737 (1985).

11) S. Tanaka, Y. Kasuya, Y. Masuda, and K. Shigenobu, J. Pharmacobio-Dyn., 6, 866 (1983).

12) M. Ohno, K. Fujita, M. Shiraiwa, A. Izumi, S. Kobayashi, H. Yoshiwara, I. Kudo, K. Inoue, and S. Nojima, J. Med. Chem., 29, 1812 (1986). 